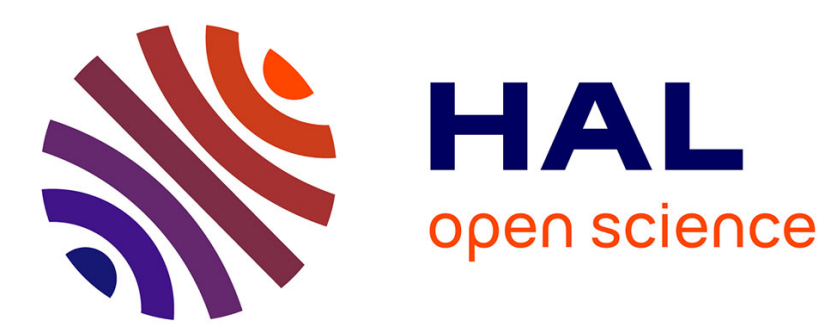

\title{
Excitation de plasmons et de phonons par battement de deux ondes électromagnétiques transversales dans un plasma
}

\author{
T. Lehner
}

\section{- To cite this version:}

T. Lehner. Excitation de plasmons et de phonons par battement de deux ondes électromagnétiques transversales dans un plasma. Revue de Physique Appliquée, 1988, 23 (9), pp.1521-1530. 10.1051/rphysap:019880023090152100 . jpa-00245980

\section{HAL Id: jpa-00245980 https://hal.science/jpa-00245980}

Submitted on 1 Jan 1988

HAL is a multi-disciplinary open access archive for the deposit and dissemination of scientific research documents, whether they are published or not. The documents may come from teaching and research institutions in France or abroad, or from public or private research centers.
L'archive ouverte pluridisciplinaire HAL, est destinée au dépôt et à la diffusion de documents scientifiques de niveau recherche, publiés ou non, émanant des établissements d'enseignement et de recherche français ou étrangers, des laboratoires publics ou privés. 


\title{
Excitation de plasmons et de phonons par battement de deux ondes électromagnétiques transversales dans un plasma
}

\author{
T. Lehner \\ Laboratoire de Physique des Milieux Ionisés, Groupe de Recherche n 29 du C.N.R.S., Ecole Polytechnique, \\ 91128 Palaiseau Cedex, France
}

(Reçu le 15 octobre 1987, accepté le 19 mai 1988)

\begin{abstract}
Résumé. - Le battement de deux ondes électromagnétiques dans un plasma pour produire une onde longitudinale (plasmon ou phonon) est réalisé expérimentalement à l'aide d'un double banc micro-onde en bande centimétrique. La détection du couplage se fait : (a) soit par une méthode d'absorption directe sur l'une des pompes ; (b) soit par diffusion hétérodyne d'une troisième onde dans des conditions de quasi-absorption. Ces méthodes de détection sont optimales : la première satisfait la relation de Manley-Rowe pour le transfert d'action entre les pompes dans le cas d'amortissement convectif ; la seconde produit une onde diffusée qui est anti-conjuguée de phase avec l'onde mère 2, dans la mesure où l'onde 3 diffère peu de l'onde mère 1.
\end{abstract}

\begin{abstract}
The beating of two transverse electromagnetic waves in a plasma on a longitudinal wave (plasmon, phonon) is demonstrated experimentally with a double microwave bench in the $\mathrm{X}$ band. The detection of the coupling is made by two methods : (a) a direct absorption method on one pump wave ; (b) an heterodyne scattering of a third wave in near absorption condition. These methods are optimal in the sense that the first one allows the detection of the whole action transfer between the pumps (Manley-Rowe relations for convective damping), while in the second case the scattered wave is the spatially antiphase conjugate of pump wave 2 for a wave 3 close to pump wave 1 .
\end{abstract}

\section{Introduction.}

La génération d'onde par mélange optique de deux ondes électromagnétiques est une méthode générale d'optique non linéaire utilisée dans différents domaines de la physique. Dans le cas d'un plasma cette méthode peut s'avérer utile dans les perspectives d'accélération collective (Tajima et Dawson) [1] en permettant la génération d'une onde longitudinale de plasma de forte amplitude. L'application aux diagnostics du plasma est également prometteuse car on peut obtenir des renseignements locaux (dans la zone d'interaction des deux ondes) sur des paramètres macroscopiques, en excitant de façon sélective une résonance du milieu choisie à l'avance (par exemple Lehner) [2].

Dans cette étude on montrera sur un cas simple comment exciter et détecter une telle onde de battement. La partie 2 expose la théorie sommaire du couplage à 3 ondes. La partie 3 donne le principe de détection, tandis que la partie 4 est consacrée aux résultats expérimentaux.
2. Théorie du couplage à trois ondes.

Le couplage à trois ondes est caractérisé ici par le battement de deux ondes en créant une troisième. On s'intéressera plus particulièrement au couplage de deux ondes transverses électromagnétiques (tem) sur une onde longitudinale (1) ou électrostatique. Ce processus est symbolisé par la notation :

$$
\text { tem }+ \text { tem } \rightarrow 1 \text {. }
$$

Le battement des deux ondes mères de fréquence et nombre d'onde $\left(k_{1}, \omega_{1}\right)$ et $\left(k_{2}, \omega_{2}\right)$ provoque un effet de force pondéromotrice modulée à la fréquence différence $\omega_{1}-\omega_{2}$. Cette force agit comme un terme source perturbant le milieu, celui-ci réagit en créant une réponse induite (onde fille à $\left(k_{\mathrm{f}}, \omega_{\mathrm{f}}\right)$ ). Le couplage est résonant lorsque $\left(k_{1}-k_{2}, \omega_{1}-\omega_{2}\right)$ satisfait à la relation de dispersion $D(k, \omega)=0$ qui est, par annulation de la constante diélectrique, la condition d'entretien d'une onde propre par le plasma.

Pour décrire l'amplitude de l'onde fille créée on 
peut utiliser les équations dites de couplage à trois modes (par exemple Weiland et Wilhelmsson) [3], qui régissent les équations d'enveloppe des trois oscillateurs :

$$
\left(\delta t+\mathbf{V}_{\mathrm{g}_{i}} \cdot \delta_{r}+\nu_{i}\right) E_{i}=C_{j k} E_{j} E_{k}^{\langle *\rangle}
$$

pour $i=1,2, \mathrm{f}$.

$E_{i}$ est l'amplitude électrique du mode $i$.

$C_{j k}$ est le coefficient de couplage à 3 ondes, $V_{\mathrm{g}_{i}}$ est la vitesse de groupe du mode $i$.

$$
\begin{aligned}
& C_{j k}=\left(\Lambda_{i j k}^{\langle 2\rangle} \cdot \hat{\mathbf{e}}_{i}:\left(\hat{\mathbf{e}}_{j} \cdot \hat{\mathbf{e}}_{k}^{\langle *\rangle}\right)\right) /(\delta D / \delta \omega) \\
& \mathbf{V}_{\mathrm{g}_{i}}=-\delta D / \delta \mathbf{k} /(\delta D / \delta \omega)
\end{aligned}
$$

$D=\operatorname{det} \mathbf{D}$, D est le tenseur dispersion du plasma.

$\Lambda_{i j k}$ est la susceptibilité d'ordre deux (tenseur d'ordre 3), $\hat{\mathbf{e}}_{i}$ est la polarisation des ondes. $\Lambda_{i j k}$ peut se calculer de façon fluide ou cinétique selon le niveau de description envisagé, $\nu_{i}$ est toute cause d'amortissement de l'onde $i$.

Lorsque $\nu_{i}$ est nul on démontre (Weibel) [4] ou (Lehner) [5] les relations de Manley-Rowe qui traduisent la variation de l'action $A=W / \omega$.

$$
\begin{gathered}
-\Delta P_{1} / \omega_{1}=\Delta P_{2} / \omega_{2}=P_{\mathrm{f}} / \omega_{\mathrm{f}} \\
\text { pour } \omega_{1}>\omega_{2}>\omega_{\mathrm{f}}
\end{gathered}
$$

$\Delta$ traduit une variation spatio-temporelle en $r, t$.

L'onde 1 est ainsi déplétée de ses photons au profit de l'onde 2 et de l'onde fille $\mathrm{f}$. La puissance $P_{\mathrm{f}}$ déposée dans l'onde fille se calcule en résolvant le système d'équations (2.1). Ceci conduit à une expression de type $P_{\mathrm{f}}=f\left(P_{1}, P_{2}\right)$ où $f$ est une fonctionnelle compliquée en général dans les cas analytiquement solubles. Cependant lorsque le champ d'interaction non linéaire (temps nécessaire à l'établissement de la saturation des amplitudes des ondes) est très long devant le temps effectif d'interaction (même condition en terme de longueur d'interaction) et si les coefficients de couplage sont suffisamment faibles, ces deux conditions étant très souvent remplies dans la pratique, on peut se contenter d'une solution linéarisée $P_{\mathrm{f}}=P_{1} P_{2} / P_{0}$, où $P_{0}$ est une puissance caractéristique du couplage considéré. $P_{0}$ peut se calculer de deux manières : directement à partir du système (2.2), ou par un calcul asymptotique. Donnons le principe de ce dernier calcul dans le cas d'un plasma infini et non magnétisé. L'équation de propagation de l'onde fille s'écrit :

$$
\begin{array}{r}
\operatorname{Rot}\left(\operatorname{Rot}\left(\mathbf{E}_{\mathrm{f}}\right)\right)+1 / c^{2} \delta / \delta t^{2} \int \mathrm{d}^{3} r^{\prime} \boldsymbol{\varepsilon}\left(\mathbf{r}, \mathbf{r}^{\prime}, t, t^{\prime}\right) \times \\
\times \mathbf{E}_{\mathrm{f}}\left(\mathbf{r}^{\prime}, t^{\prime}\right)=\mathbf{S}_{12}(\mathbf{r}, t)
\end{array}
$$

où $\varepsilon$ est la constante diélectrique (reliée à $D$ précédent). $S_{12}$ est le terme source des interactions relié au courant non linéaire par:

$$
S_{12}=-\mu_{0} \delta j\langle 2\rangle / \delta t .
$$

Ce courant est non linéaire car proportionnel aux champs électriques $E_{1}$ et $E_{2}$. Dans le cas d'un plasma uniforme $\varepsilon$ devient indépendant de la position $r^{\prime}, j^{\langle 2\rangle}$ peut se calculer de façon fluide ou cinétique.

2.1 Calcul fluide. - On se limite aux deux premières équations fluides (équations de continuité et du mouvement) :

$$
\begin{aligned}
\delta n / \delta t+\operatorname{div}(n \mathbf{V})= & 0 \\
\delta \mathbf{V} / \delta t+(\mathbf{V} \cdot \nabla) \mathbf{V}=q / m(\mathbf{E}+\mathbf{V} & \wedge \mathbf{B})+ \\
& +\nu \mathbf{V}-\nabla \mathbf{P} / n m
\end{aligned}
$$

$P$ est la pression cinétique et $\nu$ la viscosité. La force pondéromotrice est le terme source associée à la vitesse source $V\langle 2\rangle$ (par opposition aux termes induits) :

$$
\begin{gathered}
\mathbf{f}_{\mathrm{p}}=-m\left(\left(\mathbf{V}^{\langle 1\rangle} \cdot \nabla\right) \mathbf{V}^{\langle 1\rangle}\right)+ \\
+q\left(\mathbf{V}^{\langle 1\rangle} \wedge \mathbf{B}^{\langle 1\rangle}\right) \\
m \delta \mathbf{V}^{\langle 2\rangle} / \delta t=\mathbf{f}_{\mathrm{p}} \\
\mathbf{j}^{\langle 2\rangle}=q n_{0} \mathbf{V}^{\langle 2\rangle} .
\end{gathered}
$$

Avec ici $n^{\langle 1\rangle}=0$ : il n'y a pas de perturbation de densité apportée par une onde mère tem. $V\langle 1\rangle$ est solution de l'équation du mouvement linéarisée :

$$
\delta \mathbf{V}^{\langle 1\rangle} / \delta t=q \mathbf{E}^{\langle 1\rangle} / m .
$$

Dans le cas des pompes harmoniques la vitesse d'oscillation est $V\langle 1\rangle(j)=q E_{j} / m \omega_{j}$ (2.7), pour $j=1,2$. Calculant alors le champ $E_{\mathrm{f}}$ de façon induite à l'aide de l'équation (2.3), et utilisant la relation reliant $E_{\mathrm{f}}$ et $P_{\mathrm{f}}$ (ici pour une onde de type 1) on obtient l'expression [5] :

$$
\begin{aligned}
P_{\mathrm{f}}(L)=P_{1} P_{2}\left\|\boldsymbol{\Lambda}\langle 2\rangle \cdot \hat{\mathbf{e}}_{\mathrm{f}}:\left(\hat{\mathbf{e}}_{1} \cdot \hat{\mathbf{e}}_{2}^{*}\right)\right\| \times \\
\times \frac{2}{\varepsilon_{0}} A / \sum_{i=1}^{3}\left|\frac{\delta D}{\delta k}\right|_{i}
\end{aligned}
$$

$A$ est un facteur géométrique qui tient compte des sections $\sigma_{j}$ des faisceaux mères et de la longueur d'interaction $L$ entre les pompes

$$
A=\frac{\sigma_{\mathrm{f}}}{\sigma_{1} \sigma_{2}}\left|\int_{0}^{L} u_{1}(\mathbf{r}) u_{2}(\mathbf{r}) \mathrm{e}^{-i \int^{x_{0}} \Delta \mathbf{k} \cdot \mathrm{d} \mathbf{x}} \mathrm{d} x_{0}\right|^{2} .
$$

Où $\Delta k=k_{\mathrm{f}}-\left(k_{1}-k_{2}\right)$ est un désaccord éventuel en nombre d'onde, $A$ est maximale si $\Delta k$ est nul. $x_{0}$ est pris dans la direction de l'onde fille, $u_{j}(r)$ est le profil spatial du faisceau mère $j$ avec :

$$
\begin{aligned}
& \sigma_{j}=\int u_{j}(r)^{2} \mathrm{~d} \Sigma_{j} \\
& \mathbf{D}=\omega^{2}\left(\boldsymbol{\varepsilon}+\mathbf{N} \mathbf{N}-N^{2} I_{\mathrm{d}}\right) \mathbf{N}=\mathbf{k} c / \omega .
\end{aligned}
$$

Il faut noter que $\Lambda_{i j k}^{\langle 2\rangle}$ a été obtenue de façon auto- 
cohérente implicitement, car il a fallu annuler le propagateur de (2.3) et l'inverser pour trouver $E_{\mathrm{f}}$.

2.2 CAlCul CINÉtiQue. - Ce calcul non reproduit ici en détail se conduit de façon très analogue au calcul fluide: on part de l'équation de Vlasov linéarisée pour les ondes mères et on garde le terme d'ordre deux pour calculer la réponse $f^{\langle 2\rangle}$ sur la fonction de distribution. On calcule alors le courant cinétique et la densité de force à l'aide de l'expression :

$$
f_{\mathrm{p}}=\left(\rho E^{*}+j \wedge B^{*}\right)^{\langle 2\rangle} / 4+\mathrm{cc} \text {. }
$$

Le calcul auto-cohérent étant effectué à l'aide des équations de Maxwell avec les termes sources $\rho\langle 2\rangle$ et $j^{\langle 2\rangle}$ (cas de la génération d'une onde em) ou simplement avec l'équation de Poisson (cas d'une onde 1).

\subsection{APPLICATION : GÉNÉRATION D'ONDES LONGITU-} DINALES EN PLASMA NON MAGNÉTISÉ. - On va se limiter au cas expérimental où les faisceaux mères se coupent à $90^{\circ}$ l'un de l'autre, ce qui définit la géométrie du couplage avec :

$$
\begin{gathered}
\mathbf{k}_{\mathrm{f}}=\mathbf{k}_{1}-\mathbf{k}_{2} \\
\left|k_{\mathrm{f}}\right|=\left(k_{1}^{2}+k_{2}^{2}-2 k_{1} k_{2} \cos \theta\right)^{1 / 2} \text { et } \Theta=90^{\circ}
\end{gathered}
$$

$\omega_{\mathrm{f}}$ est solution de la relation $D_{L}\left(k_{\mathrm{f}}, \omega_{\mathrm{f}}\right)=0$ à résonance, sinon $\omega_{\mathrm{f}}$ est de toute façon forcée à la différence de fréquences $\omega_{1}-\omega_{2}$.

Il suffit ici de calculer le champ électrique $E_{\mathrm{f}}$ ou la fluctuation de densité associée $n f\langle 2\rangle$ pour caractériser complètement l'onde longitudinale.

2.3.1 Plasmons. - L'équation non linéaire de génération du champ électrique du mode plasma en milieu uniforme s'obtient par :

$$
\begin{array}{r}
\left(\delta / \delta t^{2}+\nu_{\mathrm{e}} \delta / \delta t+\omega_{\mathrm{pe}}^{2}-3 V_{\mathrm{e}}^{2} \Delta\right) n_{\mathrm{f}, \mathrm{e}}^{\langle 2\rangle}= \\
=-\omega_{\mathrm{pe}}^{2} / \omega^{2} \operatorname{div}\left(\mathrm{f}_{\mathrm{p}} / m_{\mathrm{e}}\right) \\
V_{\mathrm{e}}^{2}=k_{\mathrm{b}} T_{\mathrm{e}} / m_{\mathrm{e}}, \omega_{\mathrm{pe}}^{2}=n_{0} q_{\mathrm{e}}^{2} / m_{\mathrm{e}} \varepsilon_{0}
\end{array}
$$

$V_{\mathrm{e}}$ est la vitesse thermique des électrons de température $T_{\mathrm{e}}, \omega_{\mathrm{pe}}$ la fréquence de plasma et $n_{0}$ la densité moyenne. L'annulation du propagateur du membre de gauche de l'équation (2.10) conduit à la relation de dispersion $\omega(k)$ après une transformation de Fourier-Laplace. Les règles de sélection en $k$ et $\omega$ pour que le couplage soit résonant se traduisent dans le diagramme $(k, \omega)$ par une règle du parallélogramme qui est présentée avec la dispersion sur la figure 1 , pour le couplage photon-photon-plasmon

$$
\omega_{k}^{2}=\omega_{\mathrm{pe}}^{2}+3 k_{\mathrm{f}}^{2} V_{\mathrm{e}}^{2} \text {. }
$$

La densité induite a une allure lorentzienne dans

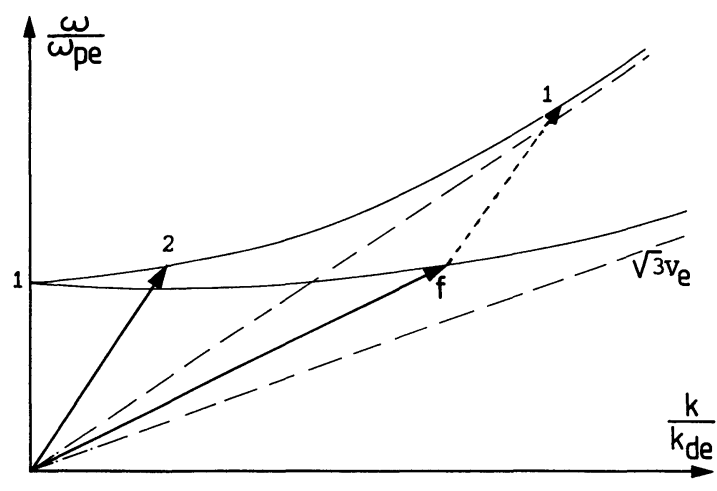

Fig. 1. - Règles de sélection pour le couplage photonphoton-plasmon.

[Sum rules for the coupling photon-photon-plasmon.]

l'espace réel, la valeur au maximum de la résonance valant :

$$
n_{\mathrm{e}}^{\langle 2\rangle} / n_{0}=\mathrm{V}(1) \cdot \mathrm{V}(2) / 4 V_{\mathrm{e}}^{2} k_{\mathrm{f}} L / 3
$$

(où $V(i)$ est donnée par (2.7)). Le facteur de qualité qui définit la largeur de la résonance vaut, dans le cas d'un amortissement convectif : $Q=\omega_{k} / \nu_{\text {c }}$, $\nu_{\mathrm{c}}=V_{\mathrm{g}_{\mathrm{f}}} / L$, soit $Q=k_{\mathrm{de}} L / 3 \quad k_{\mathrm{de}} / k_{\mathrm{f}}$, où $k_{\mathrm{de}}=\omega_{\mathrm{pe}} / V_{\mathrm{e}}$ est le nombre d'onde de Debye. $\nu_{\mathrm{c}}$ va dominer ici l'amortissement collisionnel électron-électron $\nu_{\text {e-e. }}$

2.3.2 Phonons. - Dans le cas des phonons ou ondes acoustiques ioniques l'équation de propagation s'écrit :

$$
\begin{gathered}
\left(\delta / \delta t^{2}+\nu_{\mathrm{i}} \delta / \delta t-C_{\mathrm{s}}^{2} \Delta\right) n_{\mathrm{f}, \mathrm{i}}^{\langle 2\rangle}= \\
=-\omega_{\mathrm{pe}}^{2} / \omega^{2} \operatorname{div}\left(\mathrm{f}_{\mathrm{p}} / m_{\mathrm{e}}\right) m_{\mathrm{e}} / m_{\mathrm{i}} \\
C_{\mathrm{s}}^{2}=k_{\mathrm{b}}\left(T_{\mathrm{e}}+3 T_{\mathrm{i}}\right) / m_{\mathrm{i}}
\end{gathered}
$$

$C_{\mathrm{s}}$ est la vitesse acoustique ionique. L'annulation du propagateur du membre de gauche de (2.12) conduit

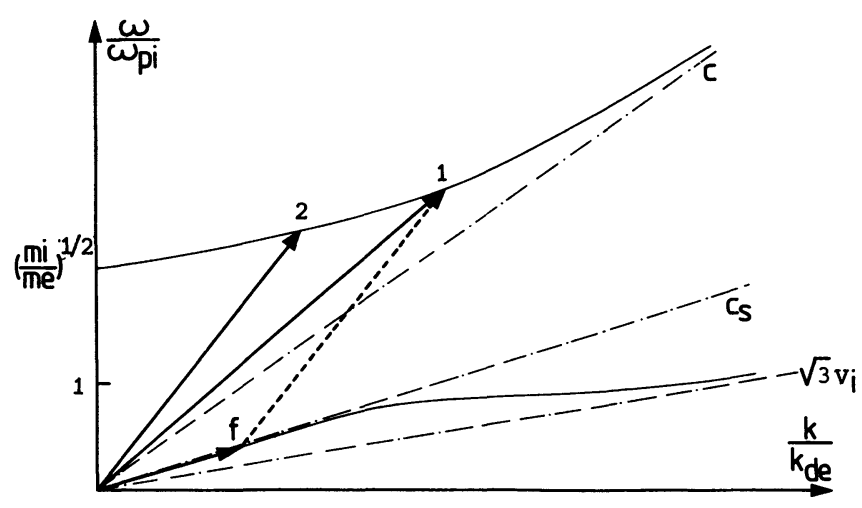

Fig. 2. - Règles de sélection pour le couplage photonphoton-phonon.

[Sum rules for the coupling photon-photon-phonon.] 
à nouveau à la relation de dispersion $\omega(k)$ et à la règle du parallélogramme, voir la figure 2 pour le couplage photon-photon-phonon.

$$
\omega_{k}^{2}=k_{\mathrm{f}}^{2} C_{\mathrm{s}}^{2} /\left(1+k_{\mathrm{f}}^{2} \lambda_{\mathrm{de}}^{2}\right) \lambda_{\mathrm{de}}=1 / k_{\mathrm{de}} .
$$

La densité induite vaut cette fois au maximum de la résonance

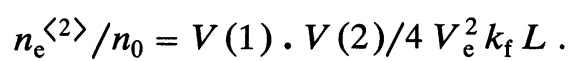

Le facteur de qualité toujours dans le cas d'un amortissement convectif est $Q=k_{\mathrm{f}} L$, lorsque l'on se place sur la partie linéaire $\omega_{k}=k C_{\mathrm{s}}$ de la dispersion. Il est environ égal au nombre de longueurs d'onde excitées dans la longueur d'interaction $L$.

Le calcul de la variation d'action $P_{\mathrm{f}} / \omega_{\mathrm{f}}$ montre que pour le cas $\Theta \simeq 90^{\circ}$ envisagé on a :

$$
P_{\mathrm{f}_{\mathrm{i}}} / \omega_{\mathrm{f}_{\mathrm{i}}} \simeq 6 P_{\mathrm{f}_{\mathrm{e}}} / \omega_{\mathrm{f}_{\mathrm{e}}} .
$$

Le nombre de «bosons" longitudinaux créables dans les deux processus est du même ordre. On donne ci-dessous l'expression de la puissance créée et sa dépendance fonctionnelle dans les paramètres du plasma et des pompes.

$$
\begin{aligned}
P_{\mathrm{f}_{\mathrm{e}}}= & P_{1} P_{2} / P_{0 \mathrm{e}} \\
P_{0 \mathrm{e}} & =\omega_{1}^{2} \omega_{2}^{2} / \omega_{\mathrm{pe}}^{4} \sigma_{1} \sigma_{2} / \sigma_{\mathrm{f}} L^{2} \omega_{\mathrm{pe}} / k_{\mathrm{f}} c V_{\mathrm{e}}^{2} / c^{2} P * / \pi \\
& P_{*}=m_{\mathrm{e}} c^{3} / r_{0}=8.7 \mathrm{GW} \\
P_{\mathrm{f}_{\mathrm{i}}} & =P_{1} P_{2} / P_{0 \mathrm{i}} \quad P_{0 \mathrm{i}} \simeq P_{0 \mathrm{e}} \omega_{\mathrm{pe}} / 6 k_{\mathrm{f}} C_{\mathrm{s}} \\
P_{\mathrm{f}_{\mathrm{e}}} & \simeq n_{0}^{3 / 2} / T_{\mathrm{e}} P_{1} P_{2} / \omega_{1} \omega_{2} 1 / \sin (\theta / 2) \\
P_{\mathrm{f}_{\mathrm{i}}} & \simeq n_{0} / T_{\mathrm{e}}^{1 / 2} P_{1} P_{2} / \omega_{1} \omega_{2} 1 / m_{\mathrm{i}}^{1 / 2} .
\end{aligned}
$$

Les valeurs numériques correspondantes seront données dans la partie 4.

\section{Théorie de la détection.}

3.1 Détection EN ABSORPTION. - On démontre que la détection en absorption sur une des ondes mères permet d'être sensible directement au transfert d'action entre les pompes [5]. Toute la puissance diffusable est alors collectée pourvu que l'on recueille toute la puissance de l'onde mère. L'avantage de la méthode est alors d'avoir une sensibilité optimale au couplage, qui est la même pour les ondes électroniques et ioniques car $P_{\mathrm{f}} / \omega_{\mathrm{f}} \simeq$ Cte. Un inconvénient pratique de la méthode est cependant que le signal est confondu avec la pompe ellemême. Pour y pallier on peut moduler en amplitude par exemple la pompe 1 et observer le transfert de modulation sur la pompe 2 quand le couplage devient résonant. Le schéma ci-dessous résume le principe d'excitation-détection; la fréquence de modulation $\Omega$ devant être inférieure à la largeur de la résonance à exciter. $\mathrm{P}$ désigne la pompe et $\mathrm{S}$ le signal.

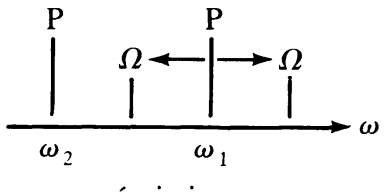

émission

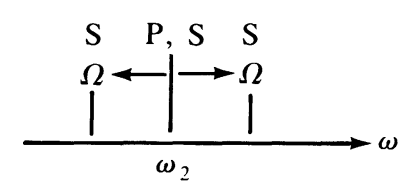

réception
Il est facile de montrer que l'on a trois raies à l'émission et à la détection :

$$
\begin{array}{lll}
\text { couple 1 } & \left(\omega_{1}-\Omega, k_{1}-\Delta k_{1}\right), & \left(k_{2}, \omega_{2}\right) \\
\text { couple 2 } & \left(\omega_{1}, k_{1}\right),\left(k_{2}, \omega_{2}\right) \\
\text { couple 3 } & \left(\omega_{1}+\Omega, k_{1}+\Delta k_{1}\right), \quad\left(k_{2}, \omega_{2}\right) .
\end{array}
$$

La diffusion de la raie $\omega_{1}-\Omega$ du couple 1 sur l'onde fille créée par le couple 3 fabrique un signal à la fréquence $\omega_{2}+\Omega$ (et à $k_{2}+\Delta k_{1}$ ), tandis que la diffusion de la raie $\omega_{1}+\Omega$ du couple 3 sur l'onde fille créée par le couple 3 donne un signal à la fréquence $\omega_{2}-\Omega$. Cette modulation autorise l'emploi d'une technique de détection synchrone nécessaire pour extraire un faible signal dans le flanc d'une porteuse d'intensité beaucoup plus élevée.

En résumé cette détection directe se fait avec des pompes qui jouent un double rôle celui de créateur de l'onde fille et de plus celui de faisceau sonde, les deux systèmes étant spatialement confondus.

3.2 DÉmONSTRATION ÉNERGÉTIQUE DES RELATIONS DE MANLEY-ROWE. - On peut faire à ce stade une évaluation pour comprendre l'amplitude obtenue dans la section suivante. Tous les champs sont supposés harmoniques en fréquence. La puissance dépensée par une des ondes peut se calculer à l'aide du théorème de Poynting ou par un calcul direct :

$$
\Delta P_{j}=\int_{V}\left\langle\mathbf{j}_{\mathrm{e}} \cdot \mathbf{E}_{j}\right\rangle \mathrm{d}^{3} \mathbf{r}
$$

où $\langle>$ désigne une valeur moyenne sur la période de l'onde $j$. Le courant $j_{\mathrm{e}}$ est ici un courant d'ordre 3 en champ électrique :

$$
\mathbf{J}_{\mathrm{e}}^{\langle 3\rangle}=\sum_{i=1,2} q_{\mathrm{e}} n f^{\langle 2\rangle}\left(\omega_{\mathrm{f}}, \mathbf{k}_{\mathrm{f}}\right) \mathbf{V}^{\langle 1\rangle}\left(\omega_{i}, \mathbf{k}_{i}\right)
$$

ou $V_{i}\langle 1\rangle$ est la vitesse d'oscillation. Le travail de ce courant permet ainsi de calculer à la fréquence $\omega_{i}=\omega_{\mathrm{f}}+\omega_{j}(i ; ⿰ j)$, et compte tenu de l'expression de $n f\langle 2\rangle$, la variation de puissance :

$$
\Delta P_{2}=\frac{\omega_{2}}{\omega_{\mathrm{f}}} P_{\mathrm{f}}, \quad \Delta P_{1}=-\frac{\omega_{1} P_{\mathrm{f}}}{\omega_{\mathrm{f}}} \quad \text { pour } \quad \omega_{1}>\omega_{2}
$$

c'est exactement la relation de Manley-Rowe. 
$J_{\mathrm{e}}^{\langle 3\rangle}$ possède aussi des composantes satellites aux fréquences $2 \omega_{i}-\omega_{j}(i \not \equiv j)$ qui peuvent, si elles induisent des champs satisfaisant à la condition de radiation, être utilisées comme diagnostic de la signature du couplage. Il est utile de remarquer que le résultat est en général d'ordre 3 dans les champs, tandis que la relation de Manley-Rowe est d'ordre 2 (cas de l'absorption).

3.3 DÉTECTION HÉTÉROdYNE A L'AIDE D'UNE TROISIĖME ONDE. - Pour s'abstraire du problème de dynamique précédent on peut songer à utiliser, une troisième onde distincte des ondes mères comme sonde. Soit une onde 3 diffusée par la fluctuation induite $n f^{\langle 2\rangle}$, ce processus obéit à l'équation d'onde

$$
\begin{gathered}
\mathbf{O}\left(r, r^{\prime}, t, r^{\prime}\right) \mathbf{E}_{4}=\mathbf{S}^{\langle 3\rangle}(\mathbf{r}, t) \\
\mathbf{S}^{\langle 3\rangle}=-\mu_{0} \delta \mathbf{j}^{\langle 3\rangle} / \delta t, \mathbf{j}^{\langle 3\rangle}=q_{\mathrm{e}} n f^{\langle 2\rangle} \mathbf{V}^{\langle 1\rangle}
\end{gathered}
$$

$V(3)$ est la vitesse d'oscillation dans l'onde 3 , et $\mathrm{O}$ est l'opérateur défini dans l'équation (2.3). Ce courant est à nouveau d'ordre trois, la résolution de (3.3) fournit le champ diffusé $E_{4}$.

Il est intéressant d'utiliser des dispositions géométriques particulières pour l'onde 3 .

$\mathrm{Si}$ on aligne l'onde 3 avec l'onde 1 dans la mesure où elle diffère peu de celle-ci (en fréquence et nombre d'onde), on va pouvoir détecter l'onde 4 dans la direction de l'onde 2 . Ceci évite des problèmes d'alignement optiques. On a en effet alors le schéma :

schéma de l'anticonjugaison de phase

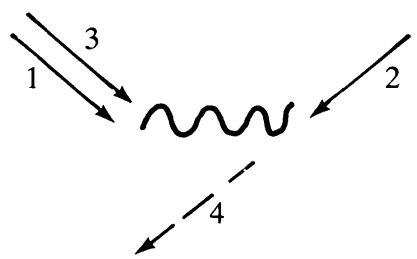

$$
\begin{array}{ll}
\text { avec } \mathbf{k}_{4}=\mathbf{k}_{3}-\left(\mathbf{k}_{1}-\mathbf{k}_{2}\right) & \omega_{4}=\omega_{3}-\left(\omega_{1}-\omega_{2}\right) \\
\text { si } \quad \mathbf{k}_{3}=\mathbf{k}_{1}+\varepsilon \mathbf{k}_{1}, & \omega_{3}=\omega_{1}+\varepsilon \omega_{1}(\varepsilon \ll 1) \\
\text { alors } \mathbf{k}_{4}=\mathbf{k}_{2}+\varepsilon \mathbf{k}_{1}, & \omega_{4}=\omega_{2}+\varepsilon \omega_{1} .
\end{array}
$$

C'est-à-dire que l'onde 4 est pratiquement anticonjuguée de phase spatialement de l'onde 2 . Un autre avantage de ce montage est que la puissance diffusée est alors d'ordre deux et non pas d'ordre trois en champ, conformément à la relation :

$P_{4} \simeq P_{\mathrm{f}} \omega_{2} / \omega_{\mathrm{f}}\left(P_{3} / P_{1}\right)^{1 / 2}$

$$
\text { à comparer à } \Delta P_{2}=P_{\mathrm{f}} \omega_{2} / \omega_{\mathrm{f}}
$$

dans le cas de l'absorption.

Une autre disposition qui n'est pas utilisée présentement mais qui le sera à l'avenir est celle de la véritable conjugaison de phase, lorsque l'onde 3 est injectée antiparallèlement à l'onde 1 (voir schéma cidessous). Dans ce cas l'amplitude diffusée est la même que celle calculée ci-dessus, mais on a exactement $k_{4}=-k_{2}$, si $k_{3}=-k_{1}$, l'onde 4 est alors la conjuguée de phase spatiale de l'onde 2 (avec $\left.\omega_{4} \simeq 2 \omega_{1}-\omega_{2}\right)$. Cette dernière méthode de détection peut être plus commode que la précédente car elle évite d'avoir à séparer l'onde 4 de l'onde 2 à la réception.

schéma de la conjugaison de phase

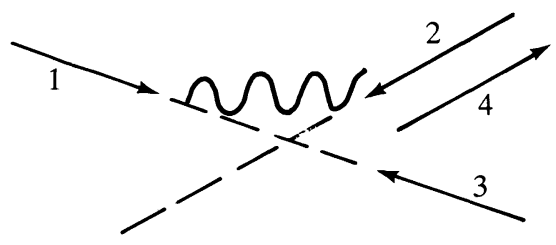

De façon générale pour améliorer la sensibilité, on aura recours à une détection synchrone en plus de la détection hétérodyne.

\section{Expériences.}

On mène l'expérience de battement dans un plasma de laboratoire. Le plasma gazeux est créée par une décharge en volume avec un confinement multipolaire aux parois. Le plasma est non magnétisé en volume, il est froid $\left(T_{\mathrm{e}} \simeq \mathrm{qqeV}\right)$, calme $\left(\delta n / n<10^{-4}\right)$, de basse densité $n_{0}<10^{12} \mathrm{~cm}^{-3}$, et uniforme à qq \% près. La figure 3 montre le schéma de l'enceinte utilisée. A cause de la densité de coupure qui est de $10 \mathrm{GHz}$, à $10^{12} \mathrm{~cm}^{-3}$, on a choisi de travailler avec un double banc de micro-ondes en bande $X(8-12,4 \mathrm{GHz})$. D'autre part les coefficients de couplage décroissent comme $1 / \omega^{2}$ et c'est le meilleur compromis compte tenu par ailleurs des problèmes d'accessibilité. Les sources sont des ORD (oscillateurs solides à résonateur diélectrique) de faible puissance $(1 \mathrm{Wcc})$ et de bonne pureté spectrale : $-80 \mathrm{dbc} / \mathrm{Hz}$ en bruit $\mathrm{fm}$ et $-120 \mathrm{dBm}$ en bruit am sont des valeurs typiques. Pour récupérer toute la puissance des ondes mères on utilise un système de coupoles elliptiques en position afocale, elles permettent un guidage efficace de la puissance des faisceaux. La figure 4 en montre le principe dans une voie : $F_{1}$ et $F_{2}^{\prime}$ sont les foyers correspondant aux cornets émetteur et récepteur et $F_{2}=F_{1}^{\prime}$ coïncide avec le centre du plasma (centre d'intersection des faisceaux).

Le succès de l'expérience dépend de la bonne stabilisation relative $\omega_{1}-\omega_{2}$ mais aussi d'une stabilisation absolue en fréquence. La figure 5 montre le synoptique des trois sources $\omega_{1}, \omega_{2}, \omega_{3}$ avec leur système d'asservissement en phase sur un quartz horloge qui sert de référence. On améliore ainsi les propriétés spectrales à long terme des sources. 


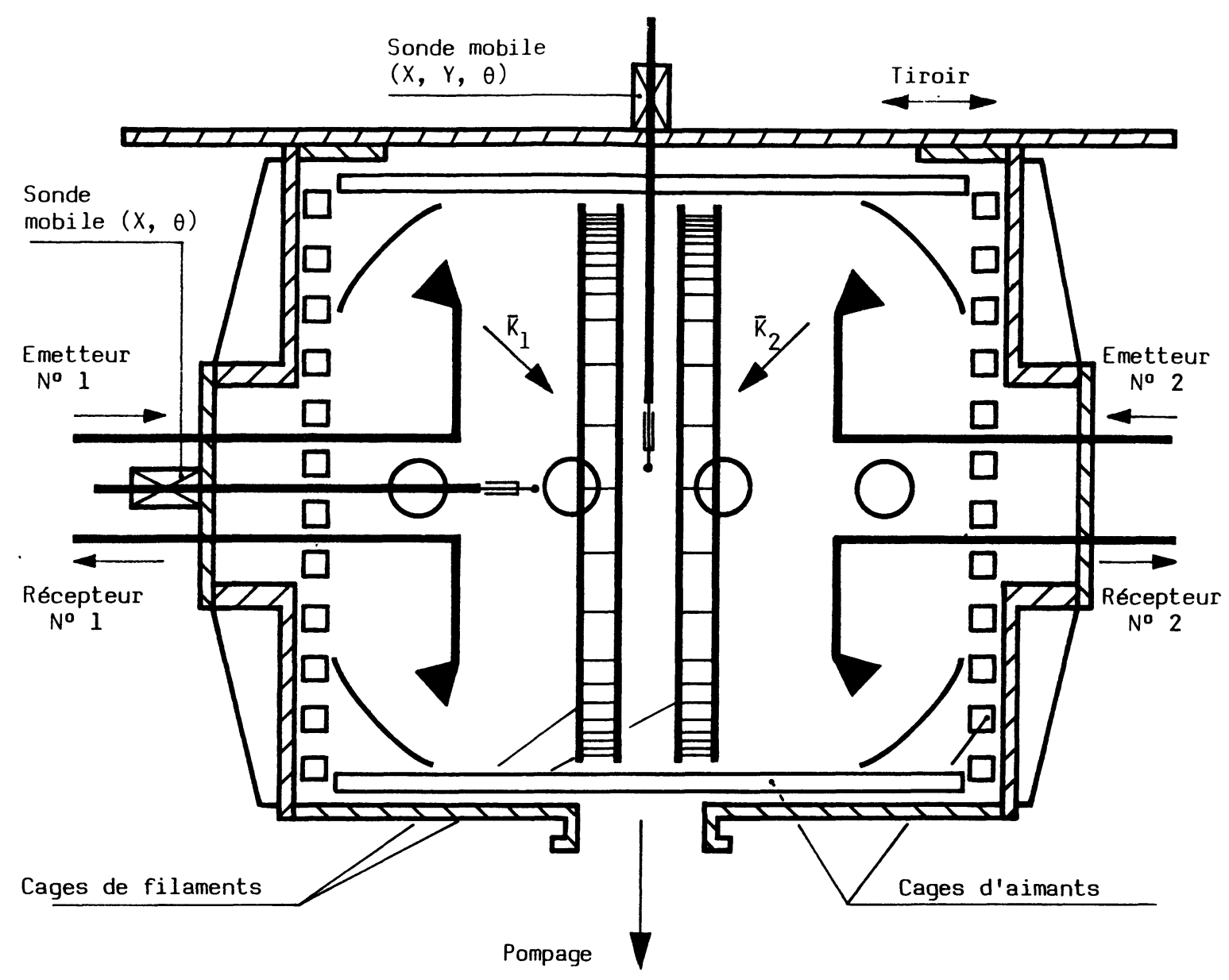

Schéma en coupe et lignes de champ

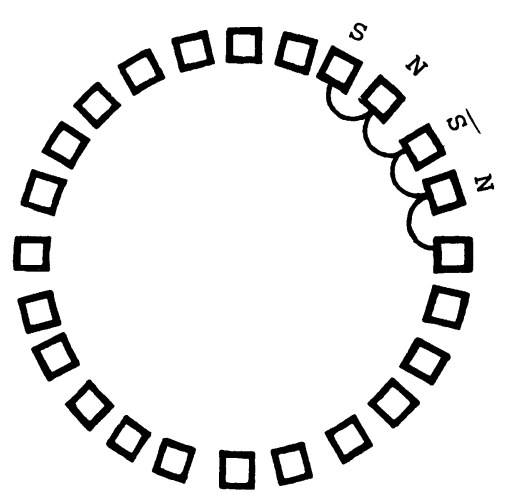

Fig. 3. - Parodie, schéma de l'enceinte à confinement multipolaire, en présence des guides et coupoles. [Schematics of the confinement device Parodie, including waveguides and elliptic mirrors.]

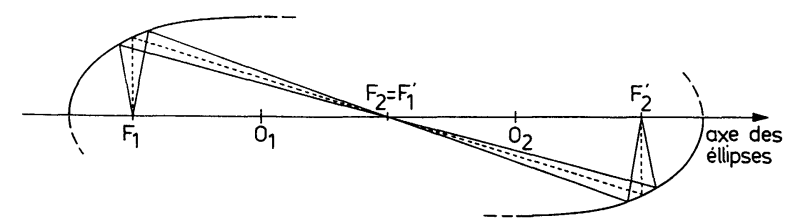

Fig. 4. - Principe de focalisation du rayonnement. [Radiation focusing principle.]
4.1 Ondes ÉlectroniQues. - Dans ce cas les conditions de l'expérience sont :

$$
\begin{gathered}
P_{1}=P_{2}=1 \mathrm{~W}, f_{1}=10 \mathrm{GHz}, f_{2}=9 \mathrm{GHz}, \\
P_{3}=0,1 \text { à } 1 \mathrm{~W}, f_{3}=10,1 \mathrm{GHz}
\end{gathered}
$$

les valeurs théoriques attendues étant au maximum, de

$$
\begin{gathered}
n^{\langle 2\rangle} / n_{0}=3 \times 10^{-6}, \quad P_{\mathrm{f}}=5 \times 10^{-8} \mathrm{~W}, \\
P_{\mathrm{f}} / \omega_{\mathrm{f}}=8 \times 10^{-18} \mathrm{~W} \cdot \mathrm{rad}^{-1} \cdot \mathrm{s}
\end{gathered}
$$




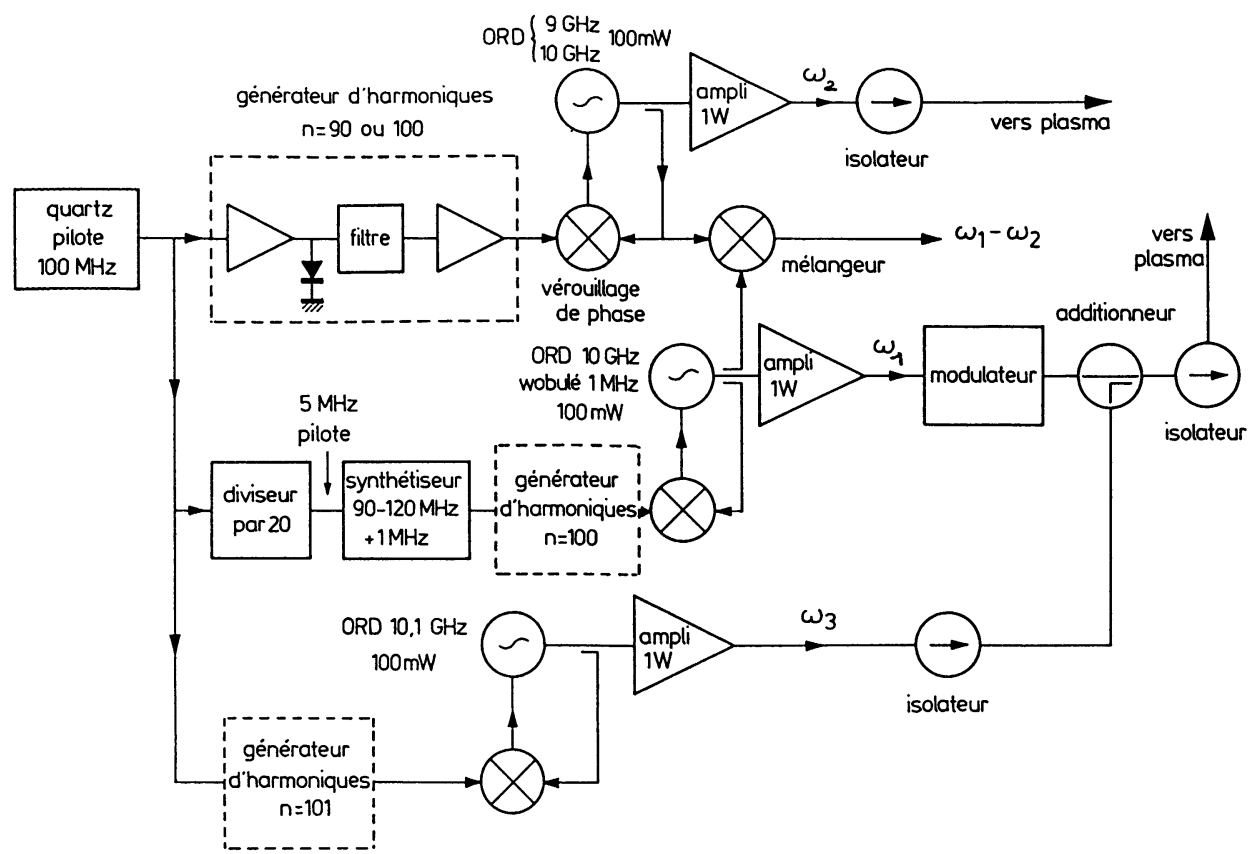

Fig. 5. - Schéma de l'électronique utilisée pour les trois sources et leur asservissement en fréquence.

[Schematics of the driving electronics : the three microwave sources and their phase locking system.]

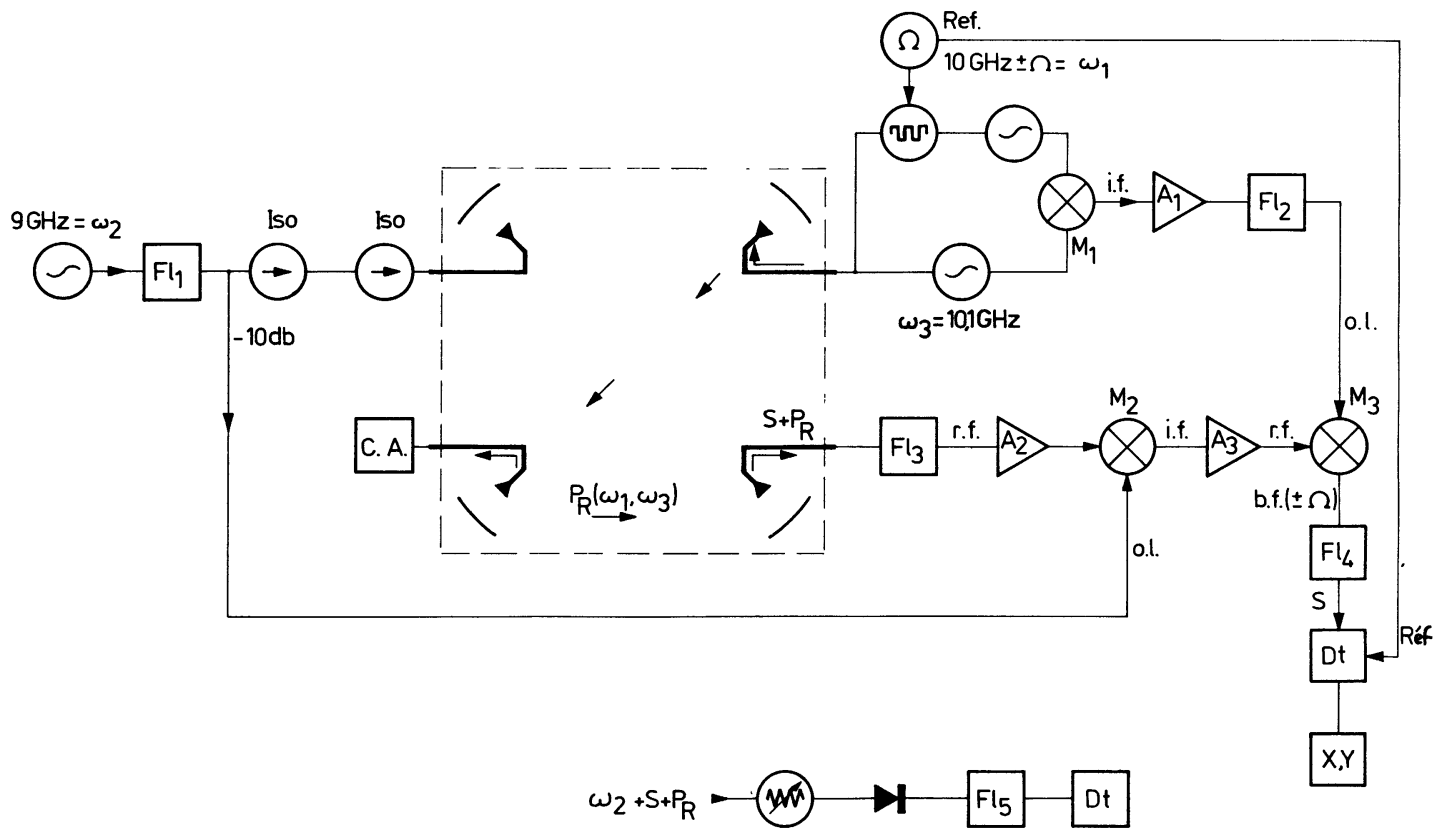

Fig. 6. - Chaîne d'excitation des ondes électroniques, détail des notations : $\mathrm{Fl}_{1}=$ filtre à cavité en coaxial centré sur $9 \mathrm{GHz}$ (bande $\mathrm{X}$ ) $; \mathrm{Fl}_{2}=$ filtre coaxial centré sur $100 \mathrm{MHz}$ (bande if) $; \mathrm{Fl}_{3}=$ filtre à cavité sur guide (ou à Yig en coaxial) centré sur $9,1 \mathrm{GHz} ; \mathrm{Fl}_{4}=$ filtre passe-bande de basse fréquence (centré sur $\Omega$ ) ; $\mathrm{Fl}_{5}=$ filtre passe-bas ; $\mathbf{M}_{1}=$ mixeur hf (bande $\left.\mathrm{X}\right) ; \mathbf{M}_{2}=$ mixeur détecteur hf (bande $\left.\mathrm{X}\right) ; \mathbf{M}_{3}=$ mixeur bf (bande if) ; $\mathrm{A}_{1}=$ amplificateur if de gain 20 ou $30 \mathrm{~dB} ; \mathrm{A}_{2}=$ amplificateur hf de gain $15 \mathrm{~dB} ; \mathrm{A}_{3}=$ amplificateur if $55 \mathrm{~dB}(+30 \mathrm{~dB}) ; P_{\mathrm{r}}=$ puissance réfléchie ; $\mathrm{S}=$ signal. En bas de la figure se trouve le cas de la détection vidéo.

[Excitation chain for electron waves, notations : $\mathrm{Fl}_{1}=$ coaxial cavity filter centered on $9 \mathrm{GHz}$ (X band); $\mathrm{Fl}_{2}=$ coaxial cavity filter centered on $100 \mathrm{MHz}$ (if band) $; \mathrm{Fl}_{3}=$ waveguide cavity filter (or coaxial Yig filter) centered on $9.1 \mathrm{GHz} ; \mathrm{Fl}_{4}=$ pass band low frequency filter (centered on $\Omega$ ) ; $\mathrm{Fl}_{5}=$ low pass filter $; \mathrm{M}_{1}=$ mixer hf $(\mathrm{X}$ band) ; $\mathbf{M}_{2}=$ mixer detector hf (X band); $\mathbf{M}_{3}=$ mixer lf (if band); $A_{1}=$ if amplifier of gain 20 or $30 \mathrm{~dB}$; $\mathrm{A}_{2}=$ hf amplifier of gain $15 \mathrm{~dB} ; \mathrm{A}_{3}=$ if amplifier $55 \mathrm{~dB}$ gain $(+30 \mathrm{~dB}) ; P_{\mathrm{r}}=$ reflected power $; \mathrm{S}=$ signal. At bottom of the figure is the video detection.] 
$n^{\langle 2\rangle} / n_{0}$ est ainsi inférieure au niveau thermique $\left(10^{-4}\right)$ des fluctuations de densité (bruit large bande). Le balayage de la résonance se fait par celui de la densité du plasma, de façon à assurer $f_{1}-f_{2} \simeq f_{\text {pe }}$ lorsque l'on passe par $1 \mathrm{GHz}(1 \mathrm{GHz}$ correspond à $n_{0}=1,22 \times 10^{10} \mathrm{~cm}^{-3}$ ). La figure 6 montre le schéma général pour l'excitation des plasmons. Le signal dans la voie 2 autour de $9 \mathrm{GHz}$ est détecté en absorption directe suivie d'une détection synchrone (bas de la figure), ou par détection hétérodyne (suivie d'une détection synchrone) à la fréquence $f_{4}=9,1 \mathrm{GHz}$, après blocage de la pompe 2 par un filtre sélectif $\left(\mathrm{Fl}_{3}\right)$ centré sur $f_{4}$. Deux étages de mélangeur-détecteur successifs $h f$ puis $b f$ ramènent le signal de $\left(f_{4}-f_{2}=100 \mathrm{MHz}\right)$ à la très basse fréquence de modulation $\Omega$. La stabilisation absolue des sources est nécessaire car les fréquences $f_{1}, f_{2}, f_{3}$ servent de référence (oscillateur local) dans les différents mélangeurs. La figure 7 montre la résonance électronique obtenue dans l'argon en fonction du courant de décharge $I_{\mathrm{d}} \simeq n_{0} \simeq f_{\mathrm{pe}}^{2}$. Le niveau de référence est celui en l'absence de modulation. La détection synchrone est faite en modulant la fréquence de la porteuse 1 en signaux sinusoïdaux à $\Omega=28 \mathrm{kHz}$. En ordonnées on porte l'amplitude linéaire, en unités arbitraires, du signal démodulé.

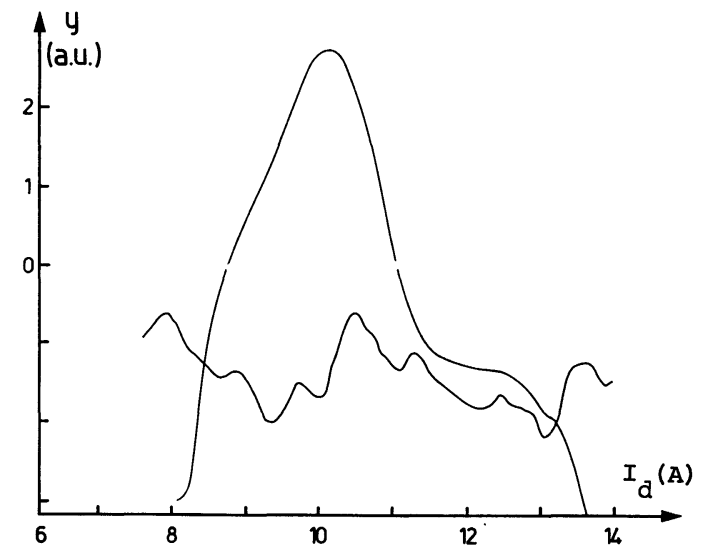

Fig. 7. - Résonance électronique dans l'argon autour de $1 \mathrm{GHz}$, obtenue par détection synchrone à $\Omega / 2 \pi=28 \mathrm{kHz}$ en modulation sinusoïdale.

[Electronic resonance in argon around $1 \mathrm{GHz}$, obtained by a lock-in detection with $\Omega / 2 \pi=28 \mathrm{kHz}$ and a sinusoidal modulation.]

La figure 8, calibrée directement en dB cette fois, montre un exemple de double résonance : en $8 \mathrm{a}$, $P_{1}=P_{2}=1 \mathrm{~W}, P_{3}=0,1 \mathrm{~W}$; en $8 \mathrm{~b}, P_{1}=0,1 \mathrm{~W}$, $P_{2}=P_{3}=1 \mathrm{~W}$. Le système d'excitation-détection est en effet symétrique : la première résonance correspond au battement à la fréquence $f_{1}-f_{2}$ diffusé par l'onde $3\left(f_{3}\right)$, alors que la seconde correspond au battement à la fréquence $f_{3}-f_{2}$ (a)
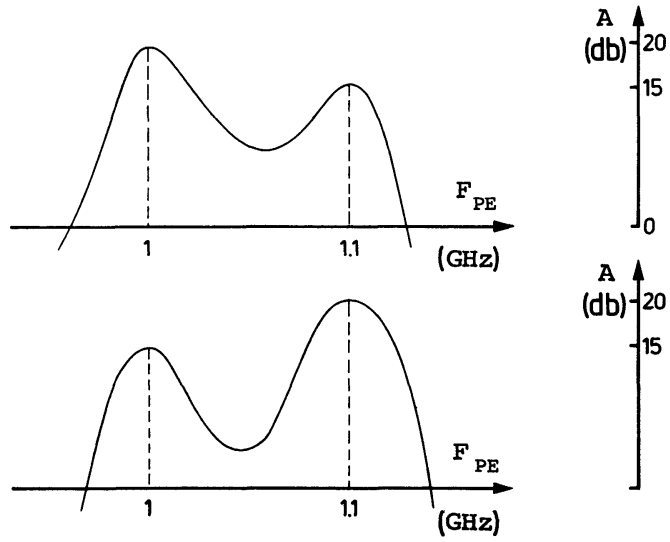

Fig. 8. - Double résonance dans l'argon autour de $1 \mathrm{GHz}$ et $1,1 \mathrm{GHz}$ obtenue avec $\Omega / 2 \pi=28 \mathrm{kHz}$.

[Double resonance in argon around $1 \mathrm{GHz}$ and $1.1 \mathrm{GHz}$ obtained with $\Omega / 2 \pi=28 \mathrm{kHz}$.]

diffusé par l'onde $1\left(f_{1}\right)$; la détection se faisant à 9,1 puis à $8,9 \mathrm{GHz}$ à l'aide d'un filtre variable.

La mesure en absorption est présentée sur la figure 9 , en présence des trois ondes $f_{1}, f_{2}$, $f_{3}$. Le détecteur le plus sensible distingue encore la double résonance qui s'estompe avec la diminution de la sensibilité des détecteurs. Les largeurs de résonance plus grandes que prévues sont dues à un effet de faible non-uniformité de la densité (voir Lehner) [6].

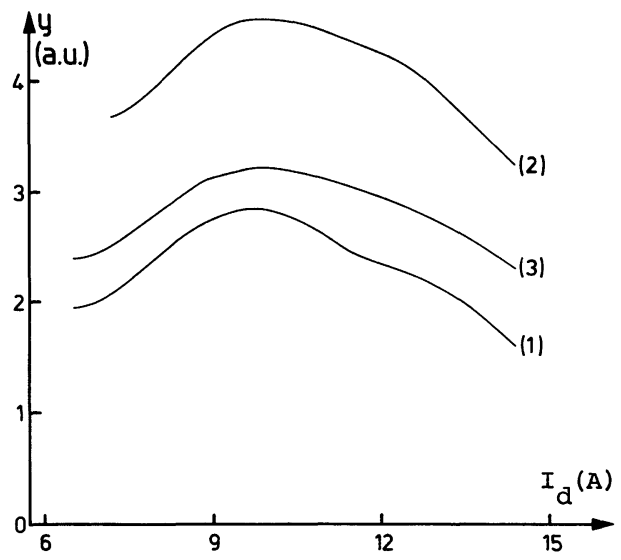

Fig. 9. - Mesure en absorption avec différents détecteurs vidéo de moins en moins sensibles : 1) sensibilité $2000 \mathrm{mV} / \mathrm{mW}$, 2) $940 \mathrm{mV} / \mathrm{mW}$, 3) $200 \mathrm{mV} / \mathrm{mW}$.

[Absorption measurement with different video detectors less and less sensible: 1) sensibility $2000 \mathrm{mV} / \mathrm{mW}$, 2) $940 \mathrm{mV} / \mathrm{mW}$, 3) $200 \mathrm{mV} / \mathrm{mW}$.]

4.2 OndES IONIQUES. - Cette fois les conditions expérimentales sont :

$$
\begin{gathered}
P_{1}=P_{2}=P_{3}=1 \mathrm{~W}, \quad f_{1}=10 \mathrm{GHz}+\Delta f(t), \\
f_{2}=10 \mathrm{GHz}, \quad f_{3}=10,1 \mathrm{GHz}
\end{gathered}
$$




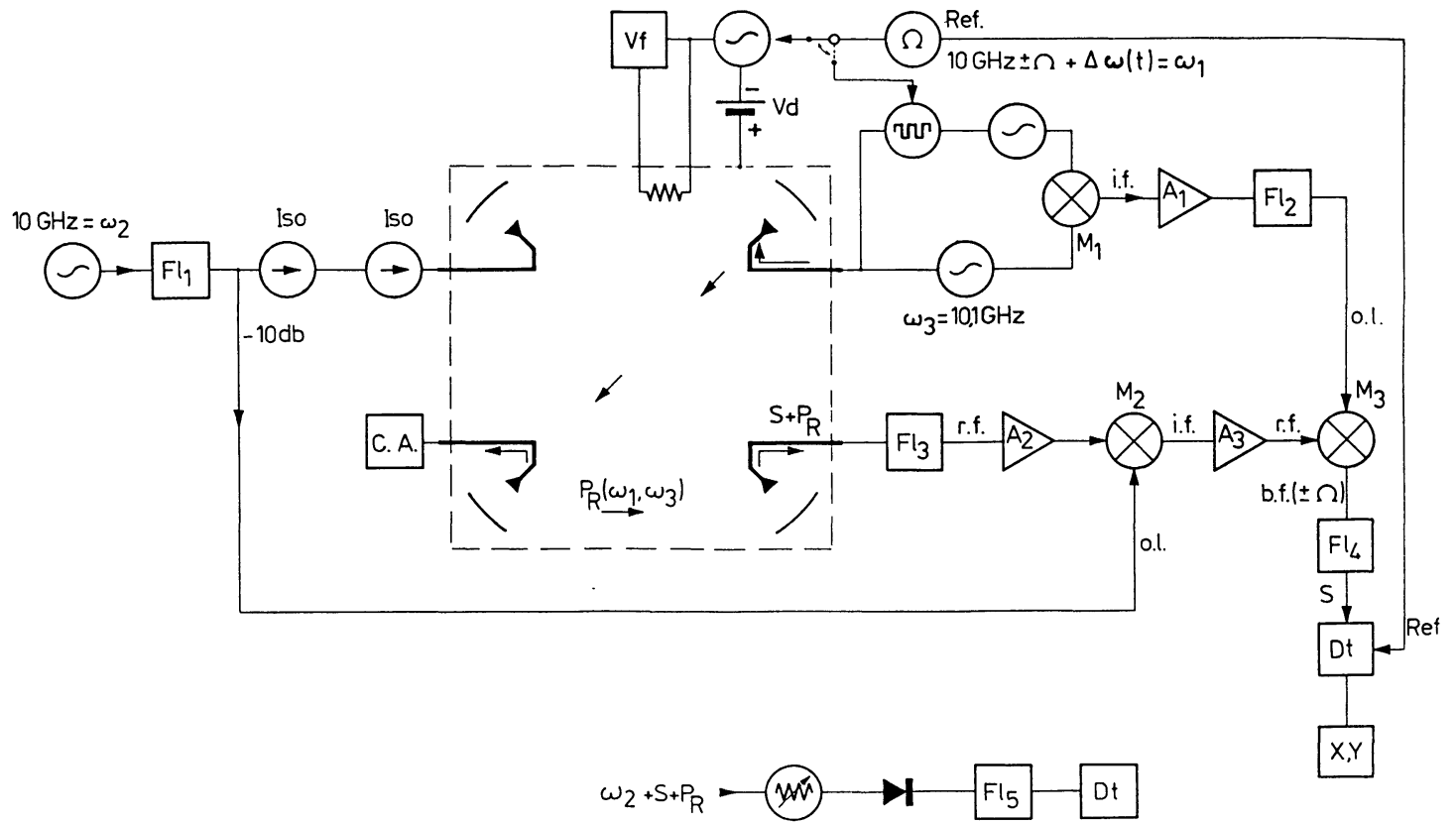

Fig. 10. - Dispositif d'excitation des ondes ioniques, les conditions sont les mêmes que sur la figure 6 sauf : $\mathrm{Fl}_{1}=$ filtre à cavité en coaxial centré sur $10 \mathrm{GHz} ; \mathrm{Fl}_{3}=$ filtre sur guide centré sur $10,1 \mathrm{GHz} ; \mathrm{A}_{3}=$ amplificateur if $(20$ à $40 \mathrm{~dB}$ selon les cas). On a figuré ici la possibilité de modulation soit de la porteuse, soit de la densité du plasma.

[Excitation device for ionic waves, conditions are the same than for figure 6 except : $\mathrm{Fl}_{1}=$ coaxial cavity filter centered in $10 \mathrm{GHz} ; \mathrm{Fl}_{3}=$ waveguide filter centered on $10.1 \mathrm{GHz} ; \mathrm{A}_{3}=$ if amplifier (20 or $40 \mathrm{~dB}$ depending on cases). Here the modulation can be done either on the pump wave, either on the plasma density.]

avec au maximum

$$
\begin{gathered}
n\langle 2\rangle / n_{0}=10^{-5}, \quad P_{\mathrm{f}}=1.4 \times 10^{-11} \mathrm{~W}, \\
P_{\mathrm{f}} / \omega_{\mathrm{f}}=5 \times 10^{-17} \mathrm{~W} \cdot \mathrm{rad}^{-1} \cdot \mathrm{s} .
\end{gathered}
$$

La figure 10 donne le schéma général d'excitation et de détection. La résonance est balayée à densité

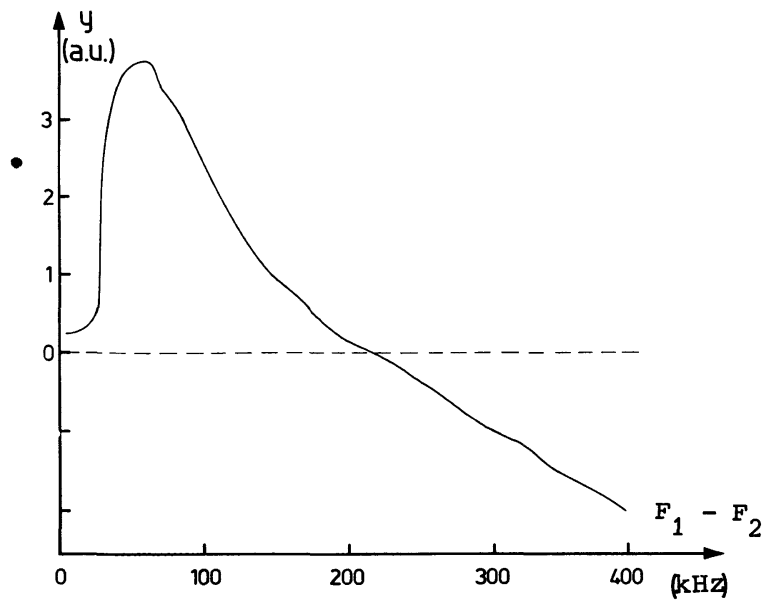

Fig. 11. - Exemple de spectre ionique dans $\mathrm{Ar}$ par modulation de la densité de plasma à $5 \mathrm{~Hz}$, détection hétérodyne puis synchrone.

[Ionic spectrum in Ar by density modulation at $5 \mathrm{~Hz}$, heterodyne detection followed by a lock-in detection technique.] fixée par mobulation en fréquence de la source 1. La modulation peut se faire soit par la densité (résultat de la Fig. 11 dans l'argon), soit par la porteuse à nouveau (Fig. 12). La valeur théorique du maximum dans l'argon est de $f_{\mathrm{i}}=k_{\mathrm{f}} C_{\mathrm{s}} / 2 \pi \simeq 75 \mathrm{kHz}$ $\left(T_{\mathrm{e}} \simeq 1 \mathrm{eV}, \quad m_{\mathrm{i}}=40 m_{\mathrm{h}}, k_{\mathrm{f}} \simeq k_{1}(2)^{1 / 2}\right)$. La valeur obtenue est inférieure à cette valeur. Ceci est due à l'existence d'une vitesse de dérive $V_{\text {de }}$ des ions qui modifie la relation de dispersion. La figure 13 mon-

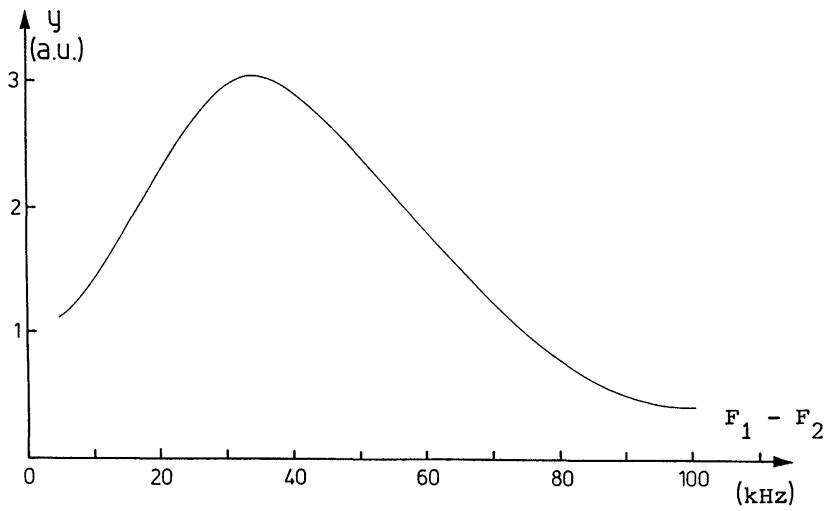

Fig. 12. - Spectre ionique dans Ar par modulation de la porteuse à $500 \mathrm{~Hz}$ en signaux carrés, détection hétérodyne puis synchrone.

[Ionic spectrum in Ar using a square wave pump modulation at $500 \mathrm{~Hz}$, heterodyne detection followed by a lockin detection technique.] 


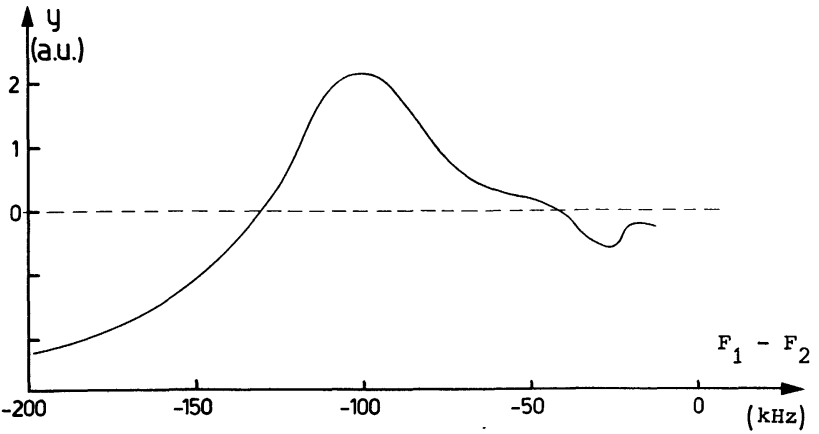

Fig. 13. - Spectre ionique mais à $f_{1}<f_{2}$ dans $\mathrm{Ar}$, mêmes conditions que pour la figure 11 .

[Ionic spectrum for $f_{1}<f_{2}$ in $\mathrm{Ar}$, same conditions than for figure 11.]

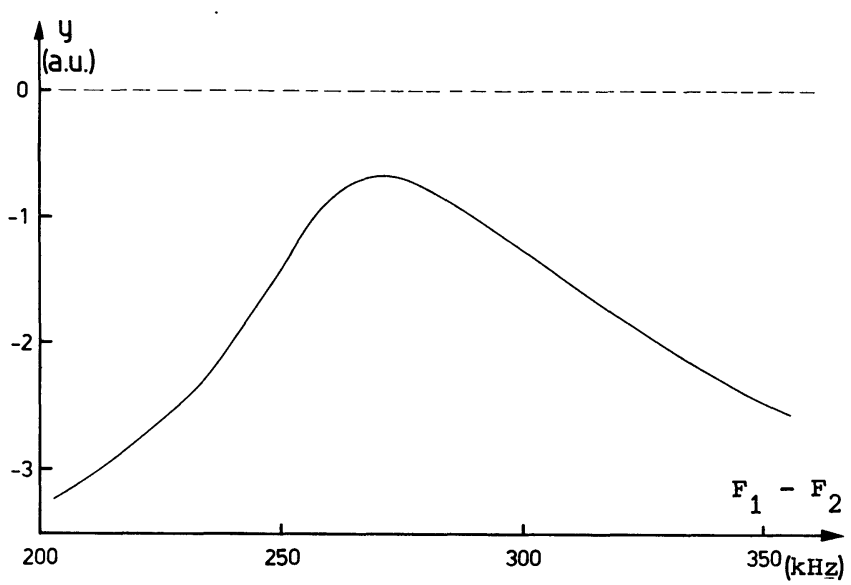

Fig. 14. - Spectre ionique dans l'hélium, mêmes conditions que pour la figure 11 .

[Ionic spectrum in $\mathrm{He}$, same conditions than for figure 11.]

tre le balayage à $f_{1}<f_{2}$ cette fois, le maximum est décalé vers les valeurs plus négatives $(\simeq-100 \mathrm{kHz})$ que $-75 \mathrm{kHz}$. Dans le cas de la figure $11, C_{\mathrm{s}}$ est remplacée par $C_{\mathrm{s}}-V_{\mathrm{de}}$ alors qu'en 13 c'est la vitesse de phase $-\left(C_{\mathrm{s}}+V_{\text {de }}\right)$ qui intervient.

La figure 14 donne la résonance dans l'hélium qui est de plus haute fréquence $\left(m_{\mathrm{i}}=4 m_{\mathrm{h}}, T_{\mathrm{e}} \simeq 3 \mathrm{eV}\right)$, que dans l'argon, à nouveau la position du pic est plus faible que prévue à cause de l'existence de

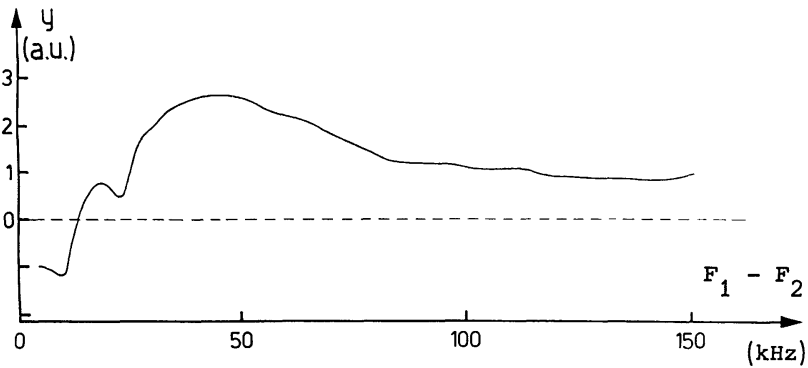

Fig. 15. - Spectre ionique mesuré en absorption dans l'Ar par modulation de la porteuse à $500 \mathrm{~Hz}$ en signaux carrés.

[Ionic spectrum measured in absorption in Ar by modulation of the pump wave at $500 \mathrm{~Hz}$ by a square wave.]

$V_{\text {de }}$. La figure 15 montre un cas d'absorption directe dans l'argon par modulation de la porteuse en signaux carrés, à $500 \mathrm{~Hz}$.

La largeur de la résonance ionique est plus large que prévue car c'est ici l'amortissement collisionnel ion-neutre qui domine l'amortissement $v_{\mathrm{c}}$ à $n_{0}$ et $T_{\mathrm{e}}$ fixées par l'expérience.

Les signaux obtenus en absorption sont toujours plus faibles qu'en détection hétérodyne car on est obligé d'atténuer la pompe et partant le signal pour ne pas saturer les détecteurs.

Une étude de calibration non détaillée ici [6] prouve que les valeurs mesurées sont compatibles avec les coefficients de couplage théoriques à condition d'inclure les différentes pertes, et elles sont aussi en accord avec la relation $P_{\mathrm{f}_{\mathrm{i}}} / \omega_{\mathrm{f}_{\mathrm{i}}} \simeq 6 P_{\mathrm{f}_{\mathrm{e}}} / \omega_{\mathrm{f}_{\mathrm{e}}}$.

\section{Conclusions.}

Nous avons montré comment exciter et détecter un couplage à trois ondes dans un cas défavorable : coefficients de couplage faibles, puissance des pompes modeste. Cependant la grande sensibilité de la détection permet de faire tout de même la mesure. Les méthodes invoquées peuvent se transposer à d'autres milieux (plasma de fusion; ionosphère, interaction laser-matière) et ne sont pas limitées au processus de couplage particulier tem-tem- 1 considéré ici.

\section{Bibliographie}

[1] Tajima, J. and Dawson, J. M., Phys. Rev. Lett. 43 (1979) 267.

[2] Lehner, T., Plasma Phys. 27 (1985) 1163.

[3] Weiland, J. and Wilhemsson, H., Coherent non linear interaction of waves in plasmas (Pergamon Press) 1977.
[4] Weibel, E. S., Phys. Fluids 19 (1976) 1237. Weibel, E. S., Phys. Rev. Lett. 37 (1976) 1619.

[5] Lehner, T., Phys. Scri. 32 (1985) 134.

[6] Lehner, T., Thèse d'état, Paris VI (1987). 